\title{
Fibroblasts prolong serum prostate-specific antigen decline after androgen deprivation therapy in prostate cancer
}

\author{
Takeshi Sasaki ${ }^{1}$, Kenichiro Ishii ${ }^{2}$, Yoichi Iwamoto ${ }^{1,3}$, Manabu Kato ${ }^{1}$, Manabu Miki ${ }^{1}$, Hideki Kanda', Kiminobu Arima ${ }^{1}$, \\ Taizo Shiraishi ${ }^{2}$ and Yoshiki Sugimura ${ }^{1}$
}

In patients with prostate cancer (PCa), serum prostate-specific antigen (PSA) is a useful marker for evaluating the effects of androgen deprivation therapy (ADT). Intuitively, most urologists expect that a more rapid PSA decline in response to ADT would be positively associated with extended survival. Recently, we have reported that prolonged gradual serum PSA decline after ADT is strongly associated with favorable prognosis in PCa patients, however, the mechanism remains unknown. We investigated the role of fibroblasts in serum PSA decline after ADT. We performed in vitro experiments using androgen-sensitive, androgen receptor (AR)-positive prostate epithelial cell lines (LNCaP, 22Rv1, and RWPE-1 cells), commercially available prostate stromal cells (PrSC), and primary cultures of prostate fibroblasts (pcPrFs). In LNCaP and 22Rv1 cells, PSA production was increased by co-culture with fibroblasts under androgen-deprived conditions. In an in vivo model using LNCaP cells, serum PSA declined rapidly after ADT becoming undetectable within 14 days in mice inoculated with LNCaP cells alone. In contrast, when LNCaP cells were co-inoculated with fibroblasts, serum PSA levels were still high on 14 days post ADT and did not drop to undetectable levels until 21 days post ADT. Tumor volumes and Ki67 labeling indices were not altered between days 14 and 21 post ADT in mice inoculated with LNCaP cells; however, those in mice inoculated with LNCaP cells plus fibroblasts decreased gradually. PSA protein was detected in all tumors on 21 days post ADT by immunohistochemical staining. Microvessel densities were higher on 14 days post ADT for tumors from mice inoculated with LNCaP cells plus fibroblasts as compared with LNCaP cells alone. In summary, co-inoculation of fibroblasts with LNCaP cells prolonged serum PSA decline after ADT and enhanced the efficacy of ADT. Prolonged serum PSA decline may indicate the presence of protective fibroblasts that preserve the AR dependence of PCa cells, improving treatment efficacy. Laboratory Investigation (2016) 96, 338-349; doi:10.1038/labinvest.2015.136; published online 7 December 2015

Prostate-specific antigen (PSA) is currently the most useful biomarker for the detection of prostate cancer (PCa). Measurement of serum PSA levels has facilitated changes in all aspects of PCa management during the past two decades. Serum PSA levels are generally proportional to tumor volume and the clinical stage of the disease. Thus, despite recognized limitations, measurement of PSA is essential for screening and monitoring of treatment response, prognosis, and progression in patients with PCa. ${ }^{1}$

Androgen deprivation therapy (ADT) is the standard systemic therapy given to patients with advanced PCa. Although ADT induces temporary remission, the majority of patients eventually progress to castration-resistant prostate cancer (CRPC), which is associated with a high mortality rate. $^{2}$ In patients with PCa undergoing ADT, PSA kinetics are considered an important indicator of the response to ADT; however, the prognostic significance of PSA kinetics remains controversial. ${ }^{3}$ Intuitively, most urologists expect that a more rapid PSA decline in response to ADT would be positively associated with extended survival. Clinical studies performed in the 1990s indicated that rapid PSA decline was associated with longer remission periods. ${ }^{4}$ However, we have recently reported interesting and counterintuitive clinical evidence. Prolonged serum PSA decline after ADT strongly indicated disease progression in patients with advanced PCa., ${ }^{5,6}$ Several recent studies have also demonstrated that longer time to PSA

Department of Nephro-Urologic Surgery and Andrology, Mie University Graduate School of Medicine, Tsu, Mie, Japan and ${ }^{2}$ Department of Oncologic Pathology, Mie University Graduate School of Medicine, Tsu, Mie, Japan

Correspondence: Professor Y Sugimura, MD, PhD, Department of Nephro-Urologic Surgery and Andrology, Mie University Graduate School of Medicine, 2-174 Edobashi, Tsu, Mie 514-8507, Japan.

E-mail: sugimura@clin.medic.mie-u.ac.jp

${ }^{3}$ Current address: Department of Urology, Mie Chuo Medical Center, Tsu, Japan.

Received 22 May 2015; revised 29 September 2015; accepted 8 October 2015 
nadir after ADT can predict favorable progression-free survival and overall survival in various hormone-naive patient populations based on large, multicenter investigations. $3,7,8$ Thus, surprisingly, a rapid decline in PSA expression after ADT strongly appears to indicate more aggressive disease. However, the mechanisms mediating this effect remain unknown. In our previous work, we found that PSA kinetics after ADT were not an accurate prognostic marker when we regarded serum PSA levels after ADT as the number of viable cancer cells. ${ }^{5,6}$

PSA is an androgen-regulated serine protease and member of the tissue kallikrein family of proteases. ${ }^{9}$ Transcription of the PSA gene is normally regulated by androgens through the androgen receptor (AR). Generally, well-differentiated PCa cells are AR dependent and PSA positive, whereas poorly differentiated PCa cells are AR independent and PSA negative. ADT treatment for patients with PCa aims to downregulate the concentration of circulating androgen or block the transcriptional activation of the AR. ${ }^{10}$ Tumor stroma surrounding cancer cells is enriched in fibroblasts secreting AR-stimulating factors, vascular endothelial growth factor (VEGF), and transforming growth factor (TGF) $\beta .{ }^{11}$ Previous studies have indicated that a number of growth factors and cytokines, including insulin-like growth factor (IGF) 1, keratinocyte growth factor (KGF; also known as fibroblast growth factor (FGF) 7), epidermal growth factor (EGF), and interleukin (IL) 6, stimulate AR signaling and PSA expression in the context of androgen deficiency. ${ }^{12}$ We have already reported that stromal remodeling after castration is accompanied by changes in the expression levels of these growth factors in the prostate. ${ }^{13}$ Importantly, most fibroblastic cells in the prostate stroma are negative for $\mathrm{AR}^{14,15}$ and the phenotypes of human PCa fibroblastic stromal cells are strongly heterogeneous. ${ }^{16}$ Several studies have reported that androgen-sensitive and -insensitive interactions between stromal and epithelial cells determine how prostate epithelial cells respond to androgen ablation. ${ }^{17,18}$ In a low-androgen environment, stromal-epithelial interactions may be an important mechanism controlling $\mathrm{AR}$ activity and AR-regulated PSA expression.

Therefore, we hypothesized that the AR-independent and heterogeneous characteristics of fibroblasts in PCa tissue could regulate the AR dependence of PCa cells, which is related to the decline in serum PSA after ADT. In this study, we investigated the role of fibroblasts in mediating the decrease in serum PSA in response to ADT. We performed in vitro and in vivo experiments using androgen-sensitive, AR-positive prostate epithelial cell lines (LNCaP, 22Rv1, and RWPE-1 cells), ${ }^{19,20}$ commercially available prostate stromal cells $(\mathrm{PrSC})$, and primary cultured prostate fibroblasts ( $\mathrm{pcPrFs}$ ) derived from three PCa patients. The effects of fibroblasts on serum PSA decline after ADT were investigated using xenograft experiments with grafting of $\mathrm{LNCaP}$ cells and fibroblasts below the renal capsule.

\section{MATERIALS AND METHODS Reagents}

Human prostate total RNA was purchased from Life Technologies, Inc. (Carlsbad, CA, USA). Dihydrotestosterone (DHT) and anti-androgen bicalutamide were purchased from Sigma-Aldrich, Inc. (St Louis, MO, USA). Recombinant human EGF, FGF2, FGF7, FGF10, hepatocyte growth factor (HGF), IGF1, TGF $\beta 1$, VEGF, and IL6 were purchased from PeproTech, Inc. (Rocky Hill, NJ, USA). Rabbit polyclonal anti-PSA, mouse monoclonal anti-neuron-specific enolase (NSE; Clone BBS/NC/VI-H14), and mouse monoclonal anti-human Ki67 antigen (Clone MIB-1) were purchased from Dako Cytomation (Copenhagen, Denmark). Rabbit polyclonal anti-AR (N-20) was purchased from Santa Cruz Biotechnology (Santa Cruz, CA, USA). Rabbit polyclonal anti-CD31 was purchased from Abcam Inc. (Cambridge, MA, USA). Matrigel was purchased from Corning Inc. (Bedford, MA, USA). Rabbit monoclonal anti-phospho-Stat3 (pStat3) and rabbit monoclonal anti-Stat3 antibodies were purchased from Cell Signaling Technology, Inc. (Beverly, MA, USA).

\section{Isolation of Fibroblasts from Prostate Tissue}

This study involving human subjects was approved by the Institutional Review Board of our institution. Consent was obtained from each patient after full disclosure of the purpose and nature of all procedures. pcPrFs were cultured from PCa specimens collected from biopsies of patients with advanced PCa. The patient characteristics are described in Table 1. Isolation of fibroblasts from specimens was performed as previously described, ${ }^{21}$ with minor modifications. Briefly, the

Table 1 Clinicopathological characteristics of patients with advanced prostate cancer

\begin{tabular}{lcccl}
\hline Patient ID & $\begin{array}{c}\text { Age } \\
\text { (years) }\end{array}$ & $\begin{array}{c}\text { Initial PSA } \\
\text { (ng/ml) }\end{array}$ & $\begin{array}{c}\text { Gleason } \\
\text { score }\end{array}$ & $\begin{array}{l}\text { Stage } \\
\text { at diagnosis }\end{array}$ \\
\hline pcPrF-M1 & 73 & 281 & $4+4$ & cT4N1M1b \\
pcPrF-M4 & 80 & 57 & $4+4$ & cT3aN0M0 \\
pcPrF-M5 & 59 & 466 & $4+4$ & cT3bN0M1b \\
pcPrF-M6 & 65 & 2298 & $4+5$ & cT4N1M1ab \\
pcPrF-M7 & 78 & 117 & $3+4$ & cT3bN0M0 \\
pcPrF-M8 & 75 & 66 & $4+5$ & cT3bN1M1a \\
pcPrF-M9 & 78 & 878 & $4+4$ & cT4N1M1b \\
pcPrF-M10 & 66 & 530 & $4+4$ & cT3bN1M1ab \\
pcPrF-M11 & 68 & 156 & $4+4$ & cT4N1M1abc \\
pcPrF-M13 & 74 & 70 & $3+4$ & cT3aN1M0 \\
pcPrF-M14 & 66 & 63 & $4+5$ & cT4N1M0 \\
pcPrF-M15 & 63 & 64 & $4+4$ & cT4N1M1b \\
\hline
\end{tabular}

ID, identification; pcPrFs, primary cultured prostate fibroblasts; PSA, prostatespecific antigen. 
tissues were cut into smaller pieces $\left(\sim 1 \mathrm{~mm}^{3}\right)$ with a knife and washed one time with $1 \times$ phosphate-buffered saline (PBS). The fragments were digested in $0.05 \%$ trypsin-ethylenediaminetetraacetic acid (EDTA) for $10 \mathrm{~min}$ at $37^{\circ} \mathrm{C}$. After digestion, the dispersed fibroblasts were cultured in medium prepared from an SCBM Bullet Kit (Lonza Group Ltd., Walkersville, MD, USA) in a T25 flask. Primary cultured fibroblastic cells were examined using real-time PCR for a number of epithelial and stromal markers, and applying a dendrogram showing stepwise characterization of fibroblastic cells from studies of Webber et al ${ }^{22}$ (Supplementary Figure 1).

\section{Cell Culture}

Androgen-sensitive, AR-positive, human prostate epithelial cell lines (LNCaP, 22Rv1, and RWPE-1 cells) were obtained from American Type Culture Collection (Rockville, MD, USA). PrSC was purchased from Lonza Group Ltd. (Basel, Switzerland). The identity of the LNCaP cells was confirmed by short tandem repeat PCR at the National Institute of Biomedical Innovation in Japan. LNCaP and 22Rv1 cells were cultured in phenol red (+) RPMI-1640 supplemented with $10 \%$ fetal bovine serum (FBS) and 1\% antibiotic/antimycotic solution. RWPE-1 cells were cultured in Keratinocyte Serum Free Medium (K-SFM) kit. PrSC and pcPrFs were cultured in medium prepared using an SCBM Bullet Kit.

\section{DHT Treatment of Cells}

LNCaP cells $\left(1 \times 10^{4}\right.$ cells/well $)$ were seeded into 96-well plates in phenol red (+) RPMI-1640 supplemented with 10\% FBS, while fibroblasts (PrSC, pcPrF-M5, pcPrF-M6, and pcPrF-M7; $8 \times 10^{3}$ cells/well) were seeded in SCBM media into 96-well plates for 1 day. The culture medium for LNCaP cells and fibroblasts was then replaced with medium supplemented with $0.5 \%$ charcoal-stripped (CS)-FBS containing various concentrations of DHT for 3 days. Cells were measured using a spectrophotometric cell counting kit (Dojindo Lab, Kumamoto, Japan).

\section{RNA Extraction and cDNA Preparation, and Real-Time PCR Analysis}

Total RNA was extracted using an RNeasy Mini Kit (Qiagen Inc., Valencia, CA, USA) in accordance with the manufacturer's instructions. The RNA concentration was determined spectrophotometrically. cDNA was reverse transcribed from $200 \mathrm{ng}$ of total RNA using a High Capacity RNA-to-cDNA Kit (Life Technologies) in a total volume $20 \mu$ l. TaqMan quantitative PCR was performed with an Applied Biosystems StepOne Real-Time PCR system (Foster City, CA, USA). TaqMan Gene Expression Assays for CHGA (Hs00900375_m1), F8 (Hs00252034_m1), KRT8 (Hs01595539_g1), DES (Hs00157258_m1), ACTA2 (Hs0042635_g1), VIM (Hs00958111_m1), COL1A1 (Hs01076777_m1), TNC (Hs01115665_m1), EGF (Hs01099999_m1), FGF2 (Hs00960934_m1), FGF7 (Hs00940253_m1), HGF (Hs00300159_m1), and IGF1
(Hs01547657_m1) were used with EagleTaq Master Mix containing ROX (Roche Diagnostics, Mannheim, Germany). All data were analyzed with StepOne Software version 2.1 (Applied Biosystems) and normalized to GAPDH (Hs02758991_g1) mRNA levels.

\section{Enzyme-Linked Immunosorbent Assay}

Serum PSA levels in mice were assayed using a PSA Enzyme Immunoassay test kit (Hope Laboratories, Belmont, CA, USA). For the quantitative determination of TGF $\beta 1$, VEGF, and IL6 protein levels, the collected aliquots of conditioned medium were subjected to ELISA using Quantikine human TGF $\beta 1$, VEGF, and IL6 immunoassay kits (R\&D Systems).

\section{Western Blot Analysis}

Subconfluent cultured LNCaP cells, PrSC, pcPrF-M5, pcPrF-M6, and pcPrF-M7 were collected by scraping, and whole-cell lysates were prepared. Extracted protein was separated by gel electrophoresis and transferred to Immobilon polyvinylidene difluoride membranes following our previously reported protocol. ${ }^{17}$

\section{Co-Culture of Prostate Epithelial Cell Lines (LNCaP, 22Rv1, and RWPE-1) with Fibroblasts}

LNCaP, 22Rv1, and RWPE-1 cells were co-cultured with each of the four fibroblast lines (PrSC, pcPrF-M5, pcPrF-M6, and pcPrF-M7) in six-well plates using cell culture inserts (BD Falcon, Franklin Lakes, NJ, USA). LNCaP, 22Rv1, and RWPE- 1 cells $\left(4 \times 10^{4}\right.$ cells/well $)$ were seeded into six-well plates in their respective recommended medium, while fibroblasts (PrSC, pcPrF-M5, pcPrF-M6, and pcPrF-M7; $2 \times 10^{4}$ cells/well) were seeded in SCBM media into cell culture inserts for 2 days. The culture medium for prostate epithelial cells and fibroblasts was replaced with phenol red (- ) RPMI-1640 supplemented with 0.5\% CS-FBS containing DHT $(0.1 \mathrm{nM})$, and the inserts with fibroblasts were then placed into six-well plates for an additional 4 days. DHT concentrations in the incubation medium were chosen based on previous studies of tissue DHT levels in recurrent PCa. ${ }^{23,24}$

\section{Growth Factor and Cytokine Stimulation of Cell Growth} Examination of the effects of growth factor and cytokine stimulation was performed as previously described, ${ }^{17}$ with minor modifications. LNCaP cells were cultured in phenol red (+) RPMI-1640 supplemented with 10\% FBS for 2 days, and the culture medium was then replaced with phenol red ( - ) RPMI-1640 supplemented with 0.5\% CS-FBS containing DHT $(0.1 \mathrm{nM})$. One day later, the culture medium was replaced with phenol red (-) RPMI-1640 containing $10 \mathrm{ng} / \mathrm{ml}$ each of recombinant EGF, FGF2, FGF7, FGF10, HGF, IGF1, TGF $\beta 1$, VEGF, and IL6. Cells were then incubated for 4 days before analysis. 


\section{Animal Studies}

All animals were maintained in a pathogen-free environment, under experimental protocol guidelines approved by the Mie University's Committee on Animal investigations. Male athymic nude mice (BALB/c, nu/nu, 6-8 weeks old) were used for all the experiments. Mice were purchased from CLEA Japan, Inc. (Tokyo, Japan).

\section{In Vivo Xenograft Model}

Subconfluent cultures of LNCaP cells and fibroblasts (PrSC, pcPrF-M5, pcPrF-M6, and pcPrF-M7) were trypsinized and counted. Xenografts without fibroblasts contained $5 \times 10^{5}$ LNCaP cells. Xenografts with fibroblasts were prepared by mixing $2.5 \times 10^{5}$ LNCaP cells and $2.5 \times 10^{5}$ fibroblasts in suspension. Pelleted cells were resuspended in $50 \mu \mathrm{l}$ neutralized type I rat tail collagen gels and then grafted beneath the renal capsule of male athymic mice (6-8 weeks old). In total, $1 \times 10^{6}$ LNCaP cells were grafted in each mouse. For the androgen deprivation experiment, mice were randomized on 14 days post transplantation. Mice treated with ADT were castrated and orally administered a bicalutamide $(25 \mathrm{mg} / \mathrm{kg})$ suspension with $0.5 \%$ carboxymethylcellulose in a 5 -days-on/ 2-days-off schedule through a 22-gauge gavage needle; the control group underwent sham operation and received the diluent. Mice were killed on 14 and 21 days post ADT. Tumor weights and serum PSA levels were measured.

\section{Histopathology and Immunohistochemistry}

For histopathology, standard hematoxylin and eosin staining was carried out. Next, immunohistochemical staining was performed with an ImmPRESS Reagent Kit (Vector Laboratories, Inc., Burlingame, CA, USA). Antigen retrieval was performed using $10 \mathrm{mM}$ sodium citrate buffer (pH 6.0) for AR, NSE, Ki67, and CD31. Antigen Unmasking Solution (Vector Laboratories, Inc.) was used for PSA. The antigen-antibody reaction was visualized using 3'3-diaminobenzidine tetrahydrochloride as a substrate. Sections were counterstained with hematoxylin and examined by light microscopy.

Cell proliferation in tumors was determined by the percentage of Ki67-positive nuclei in 10 different areas at $\times 400$ magnification from each tissue specimen. A 'microvessel' was defined as mouse-specific CD31-positive endothelial cells that formed a vascular lumen. The number of microvessels was counted in 10 different areas at $\times 200$ magnification from each tissue specimen.

\section{Matrigel Plug Assay}

Fibroblasts (PrSC, pcPrF-M5, pcPrF-M6, and pcPrF-M7) were mixed with Matrigel and injected subcutaneously into the flanks of nude mice (BALB/c, nu/nu, 6-8 weeks old) at a density of $1 \times 10^{5}$ cells $/ 0.4 \mathrm{ml}$. As controls for this assay, mice were injected with $0.4 \mathrm{ml}$ Matrigel alone. For the androgen deprivation experiment, mice were randomized at 10 days after transplantation using the same method as the in vivo xenograft model. On day 7 post ADT, the mice were perfused with PBS containing $2 \mathrm{mM}$ EDTA for $40 \mathrm{~min}$ after intravenous injection with $1 \%$ Evans blue dye. The amount of Evans blue eluted with formamide from the Matrigel plugs was measured with a spectrophotometer $(620 \mathrm{~nm})$, as previously described. ${ }^{25}$

\section{Statistical Analysis}

Data are presented as the mean \pm s.d. Statistically significant differences between two groups were determined using Student's $t$-test. For comparisons among more than two groups, Tukey or Dunett's multiple comparison tests were used. Differences with $P$-values of $<0.05$ were considered statistically significant. All statistical analyses were performed using SPSS Statistics version 22.0 (IBM Corp.).

\section{RESULTS}

\section{Characteristics of Primary Cultured Prostate Fibroblasts In Vitro}

Fibroblasts expressed mRNA of stromal markers such as TNC, COL1A1, and ACTA2 (Figure 1a). In pcPrFs, expression levels of TNC, COL1A1, FGF2, FGF7, and IGF1 mRNA and secreted TGF $\beta 1$ protein were generally higher than those in PrSC (Figures $1 \mathrm{~b}$ and $\mathrm{c}$ ). However, the expression levels of ACTA2, EGF, and HGF mRNA, and secreted VEGF and secreted IL6 protein were variable in different pcPrFs. pcPrF-M5, pcPrF-M6, and pcPrF-M7 were selected for the subsequent experiments because of their diverse expression pattern of secreted IL6, TGF $\beta 1$, and VEGF. As illustrated in Figure 1d, pcPrF-M5 and pcPrF-M6 showed an expanded and flattened cell shape as compared with PrSC and pcPrF-M7 cells. AR protein was not detected in PrSC, pcPrF-M5, pcPrF-M6, or pcPrF-M7 (Figure 1e). DHT stimulated AR-positive LNCaP cell proliferation in a concentration-dependent manner, but did not stimulate AR-negative fibroblasts (PrSC, pcPrF-M5, pcPrF-M6, and pcPrF-M7; Figure 1f).

\section{Effects of Co-Culture with Fibroblasts on LNCaP, 22Rv1, and RWPE-1 Cells In Vitro}

Cell proliferation of LNCaP cells was significantly increased when cells were co-cultured with $\mathrm{PrSC}$ or pcPrFs (Figure 2a; $P<0.01$ ). The expression levels of AR and PSA were increased in LNCaP and 22Rv1 cells co-cultured with PrSC or pcPrFs, but not in RWPE-1 cells (Figure 2b and Supplementary Figure 2). NSE proteins and phosphorylation of Stat3 were increased in LNCaP cells co-cultured with PrSC or pcPrFs (Figure 2b). In LNCaP cells, no changes in Akt phosphorylation were observed, and extracellular signal-regulated kinase (ERK) phosphorylation was not apparent in co-cultured cells (data not shown).

\section{Effects of Growth Factors and Cytokines on LNCaP Cells In Vitro}

The proliferation of LNCaP cells was significantly increased by EGF and IGF1 (Figure 2c; $P<0.01$ ). The expression levels of AR, PSA, and NSE proteins in $\mathrm{LNCaP}$ cells were increased 


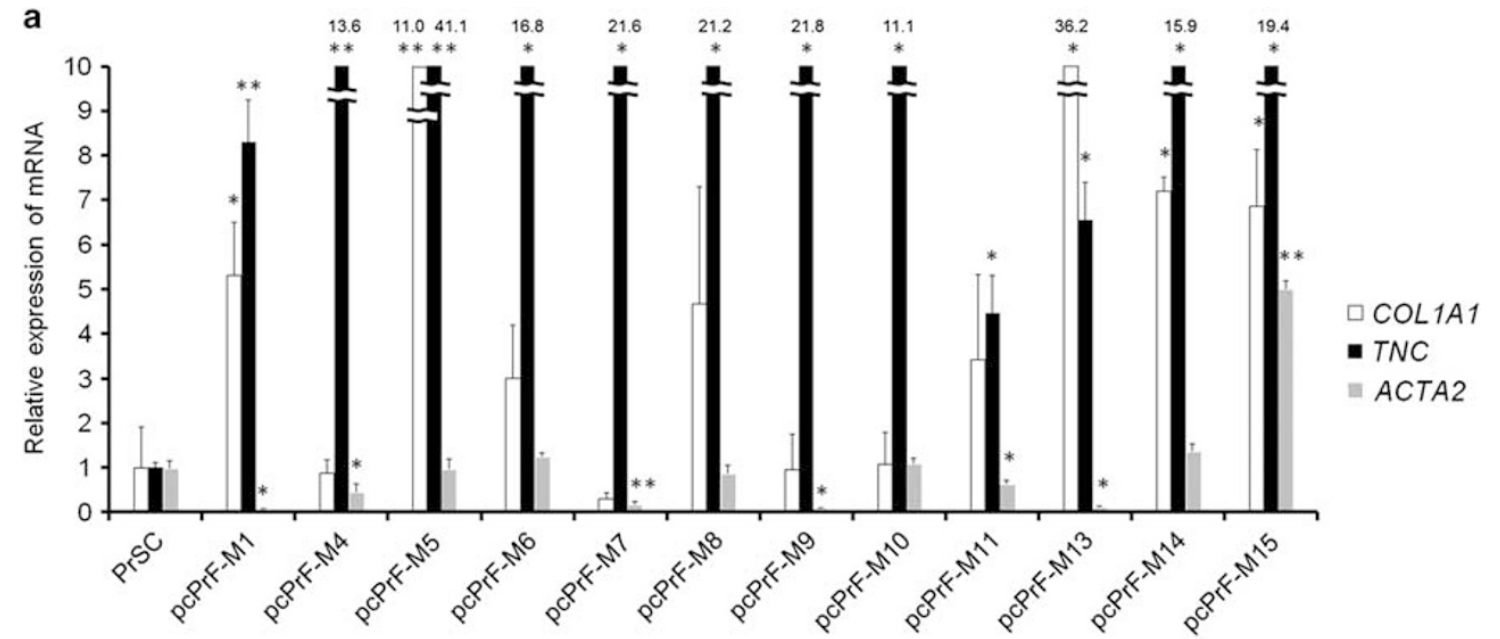

b
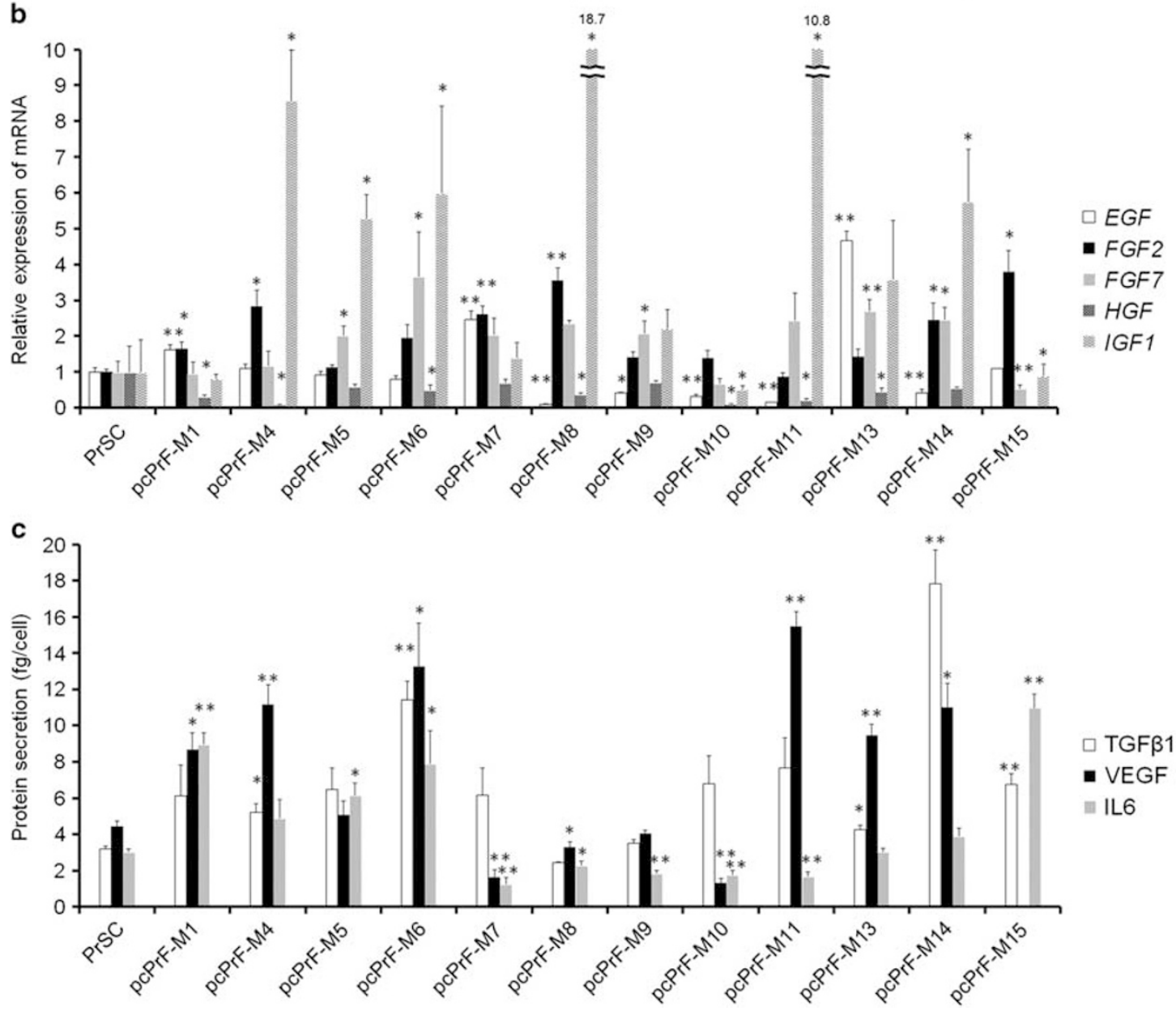

Figure 1 Characteristics of human primary cultured prostate fibroblasts in vitro. Total RNA was isolated from cells and subjected to TaqMan quantitative RT-PCR analysis. The relative expression of mRNA for stromal markers (COL1A1, TNC, and ACTA2) (a) and growth factors (EGF, FGF2, FGF7, HGF, and IGF1) (b) were determined in fibroblasts. (c) Secretion of TGF $\beta 1$, VEGF, and IL6 from fibroblasts. For the quantitative determination of TGF $\beta 1$, VEGF, and IL6, aliquots of conditioned medium were subjected to ELISA. ${ }^{*} P<0.05$, ${ }^{*} P<0.01$ vs PrSC. AR independence of primary cultured fibroblasts in vitro. (d) Cell morphology of PrSC, pcPrF-M5, pcPrF-M6, and pcPrF-M7. Bar $=100 \mu \mathrm{m}$, magnification $=\times 200$. (e) Western blot analysis for AR. Equal amount of cell lysates from growing cultures of LNCaP cells, PrSC, pcPrF-M5, pcPrF-M6, and pcPrF-M7 were subjected to western blotting. Equal loading was confirmed by detection of actin. AR protein from LNCaP cells was used as a positive control. (f) Effects of DHT on the proliferation of LNCaP cells, PrSC, pcPrF-M5, pcPrF-M6, and pcPrF-M7. ${ }^{*} P<0.05,{ }^{* *} P<0.01$ vs the untreated control. AR, androgen receptor; DHT, dihydrotestosterone; VEGF, vascular endothelial growth factor. 
d

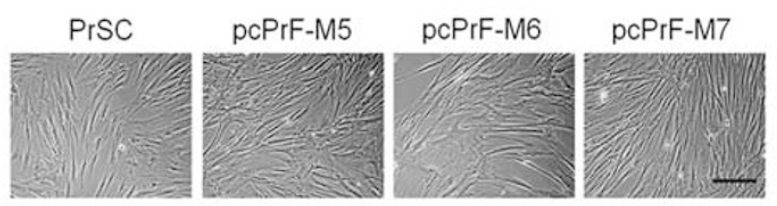

e

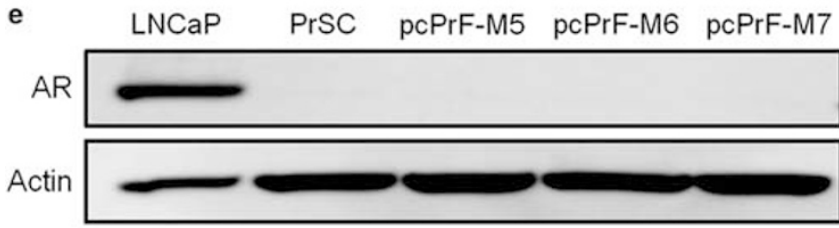

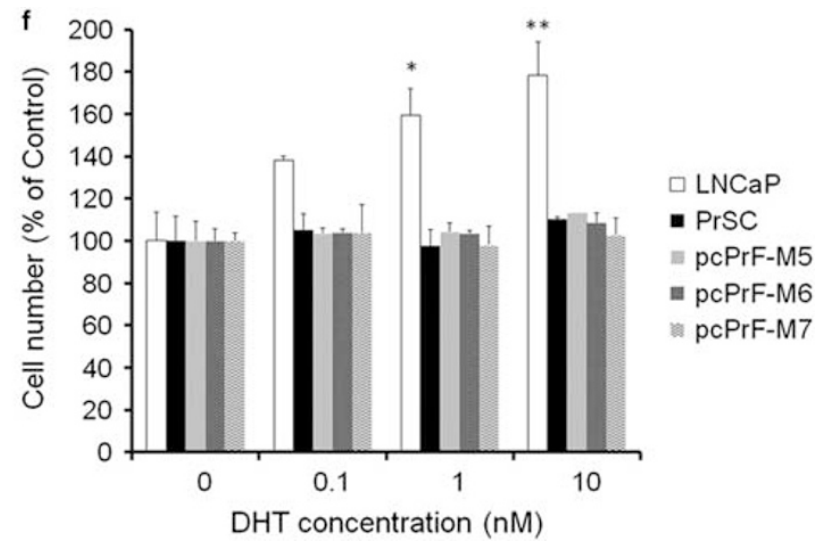

Figure 1 Continued.

by EGF, IGF1, and IL6 (Figure 2d). In addition, Stat3 phosphorylation was increased by treatment with IL6, but not with EGF or IGF1 (Figure 2d). Phosphorylation of ERK was increased by treatment with EGF, but not with IGF1 or IL6 (data not shown). No changes in the phosphorylation of Akt were observed (data not shown).

\section{Effects of ADT on the Decline in Serum PSA and Histopathological Characteristics of Xenografts Derived from Co-Inoculation of LNCaP Cells with Fibroblasts In Vivo}

Serum PSA titers in mice inoculated with LNCaP cells alone rapidly declined following $\mathrm{ADT}$. In contrast, that in mice inoculated with LNCaP cells plus fibroblasts decreased gradually (Figure 3a), i.e., serum PSA levels on day 14 post ADT were maintained by co-inoculation with fibroblasts. Interestingly, the various fibroblasts exerted different effects on the maintenance of serum PSA levels (Figure 3a). In addition, tumor volumes and Ki67 labeling indices were not altered between days 14 and 21 post ADT in mice inoculated with LNCaP cells; however, those in mice inoculated with LNCaP cells plus fibroblasts decreased gradually (Figures $3 \mathrm{~b}$ and c), i.e., the effects of ADT between days 14 and 21 post ADT were maintained by co-inoculation with fibroblasts. As shown in Figure 4a, all tumors grown in mice treated with ADT showed reduced tumorigenesis as compared with those in intact mice. AR and PSA proteins were still expressed in all tumors at day 21 post ADT, even if serum PSA levels were very low or undetectable (Figure 4a). NSE staining was diffuse among ADT-treated and intact hosts on day 21 post ADT (Figure 4a). Microvessel density (MVD) in LNCaP cells alone and + pcPrF-M7 rapidly declined; in contrast, that in + PrSC, + pcPrF-M5, and +pcPrF-M6 slowly declined (Figure $4 \mathrm{~b}$ and Table 2).

\section{Effects of Fibroblasts on Angiogenesis In Vivo}

To further address the functional role of fibroblasts in mediating angiogenesis, we performed in vivo Matrigel plug assays. Figure 5 a revealed the characteristic gross appearance of Matrigel combined with fibroblasts (PrSC, pcPrF-M5, pcPrF-M6, and pcPrF-M7). Evans blue was used to quantify the extent of angiogenesis. Angiogenesis was significantly increased in the presence of Matrigel plus PrSC or pcPrFs compared with that in the presence of Matrigel alone (Figure 5b; $P<0.01$ ). In addition, angiogenesis was significantly increased in the presence of Matrigel plus pcPrF-M5 or pcPrF-M6 compared with that in the presence of Matrigel plus PrSC or pcPrF-M7 (Figure 5b; $P<0.01$ ). Importantly, fibroblast-induced angiogenesis was not affected by ADT (Figure 5b).

\section{DISCUSSION}

The major finding in this study was that the presence of AR-independent fibroblasts resulted in a prolonged decline in serum PSA after ADT and enhanced the efficacy of ADT as measured by tumor volume and $\mathrm{Ki} 67$ index in an in vivo system. We also demonstrated that fibroblasts had two mechanisms for regulating serum PSA decline after ADT: (1) maintenance of microvessels in the tumors; and (2) secretion of AR-stimulating soluble factors. Fibroblasts have diverse capacities for neovascularization and varying expression patterns of these soluble factors. The soluble growth factors EGF and IGF1, as well as the cytokine IL6, stimulated the expression of AR and NSE protein in LNCaP cells in vitro. Importantly, the expression of NSE in PCa cells could transform cells from an AR-dependent state to an AR-independent state. On the basis of our data, we were able to form a hypothesis to explain our previous clinical observations. ${ }^{5,6}$ Figure 6 illustrates this concept and its suggested role for fibroblasts after ADT in PCa patients. First, 'protective' fibroblasts prolong the duration of blood supply in the tumors. Second, 'protective' fibroblasts cause persistent stimulation of AR in PCa cells, preventing acute loss of AR function in PCa cells. Thus, protective fibroblasts exhibiting prolonged serum PSA decline may preserve the AR dependence of PCa cells after ADT. This would decrease the selective pressure that might lead to the preferential selection of more aggressive epithelial phenotypes. Moreover, these 

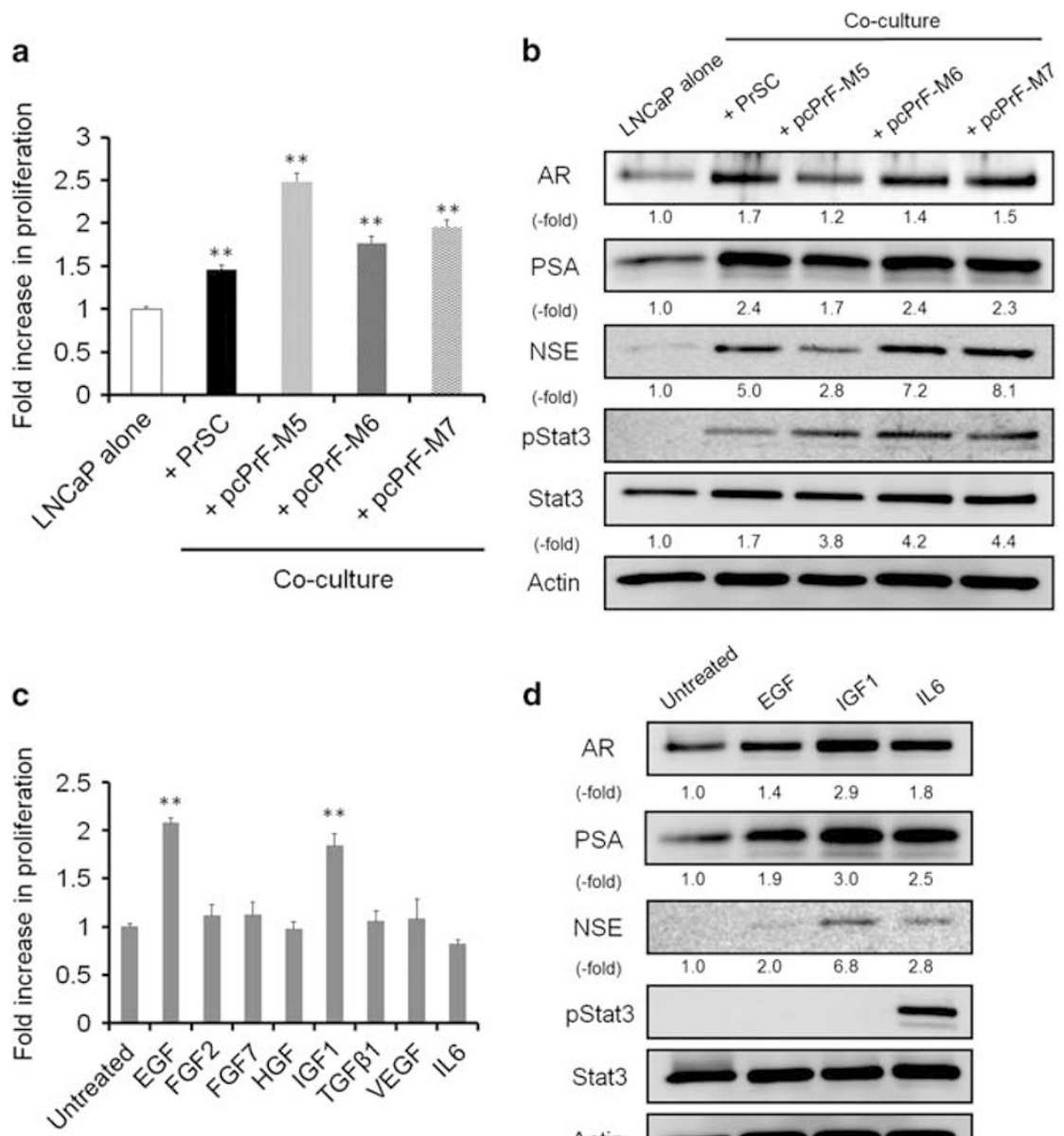

d
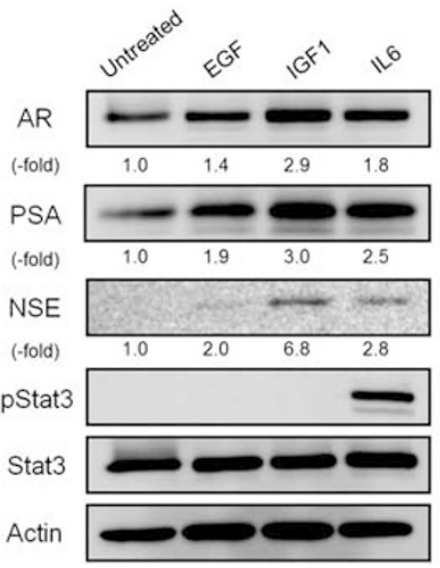

Figure 2 Effects of co-culture with fibroblasts on PSA expression in LNCaP cells in vitro. (a and $\mathbf{b}$ ) LNCaP cells were co-cultured with fibroblasts using cell culture inserts for 4 days in phenol red (-) RPMI-1640 with $0.5 \%$ CS-FBS containing DHT ( $0.1 \mathrm{nM})$. (a) Cell proliferation. ${ }^{* *} P<0.01$ vs LNCaP cells alone. (b) Cell lysates from growing cultures of co-cultured LNCaP cells were subjected to western blotting and probed with antibodies against each protein. Protein levels were compared using actin as a loading control. (c and d) LNCaP cells were treated with $10 \mathrm{ng} / \mathrm{ml}$ of each growth factor and cytokine for 4 days in phenol red (-) RPMI-1640 with 0.5\% CS-FBS containing DHT (0.1 nM). (c) Cell proliferation. ${ }^{* *} P<0.01$ vs untreated control. (d) Cell lysates from growing cultures of LNCaP cells were subjected to western blotting and probed with antibodies against each target protein. Protein levels were compared with actin loading controls. AR, androgen receptor; DHT, dihydrotestosterone; PSA, prostatespecific antigen.

fibroblasts could enhance treatment efficacy, resulting in a more favorable prognosis.

Acute loss of AR function after ADT is not only associated with apoptosis and reduction of PSA secretion in PCa cells but also triggers AR-independent growth. Disruption of androgen signaling by ADT may result in the deregulation of cell cycle control, which could contribute to carcinogenesis. ${ }^{26}$ Nelson et al provided four molecular-state frameworks for $\mathrm{AR}$ activation in PCa after $\mathrm{ADT}$ as follows: state $1=$ endocrine androgen dependent and AR dependent; state $2=$ intracrine androgen dependent and AR dependent; state $3=$ androgen independent and AR dependent; and state $4=$ androgen independent and $\mathrm{AR}$ independent. ${ }^{27}$ State 4 is considered the fatal stage, at which AR signaling is abolished and neuroendocrine (NE) differentiation occurs. Burchardt et al reported that $\mathrm{LNCaP}$ cells inoculated into castrated mice induced a significant increase in NE cells compared with those in intact mice. ${ }^{28}$ Although the mechanism of $\mathrm{NE}$ transdifferentiation induced by androgen deprivation of AR-dependent PCa cells has been investigated, the mechanism mediating NE differentiation is still not clear. ${ }^{29}$ Our data demonstrated that fibroblasts stimulated proliferation and AR expression in $\mathrm{PCa}$ cells under androgen deprivation in vitro. Moreover, in vivo, tumor volumes and Ki67 labeling indices were significantly higher in tumors from mice inoculated with LNCaP cells plus fibroblasts compared with those in tumors from mice inoculated with LNCaP cells alone. However, long-term reductions in tumor volumes and Ki67 labeling indices were observed after ADT in mice inoculated with LNCaP cells plus fibroblasts, but not in mice inoculated with LNCaP cells alone. These data appear to be contradictory; however, with regard to the framework of $\mathrm{PCa}$, fibroblast-dependent AR stimulation of $\mathrm{PCa}$ cells would maintain cells at stage 3 , which is considered curable using 


\section{Co-inoculation}
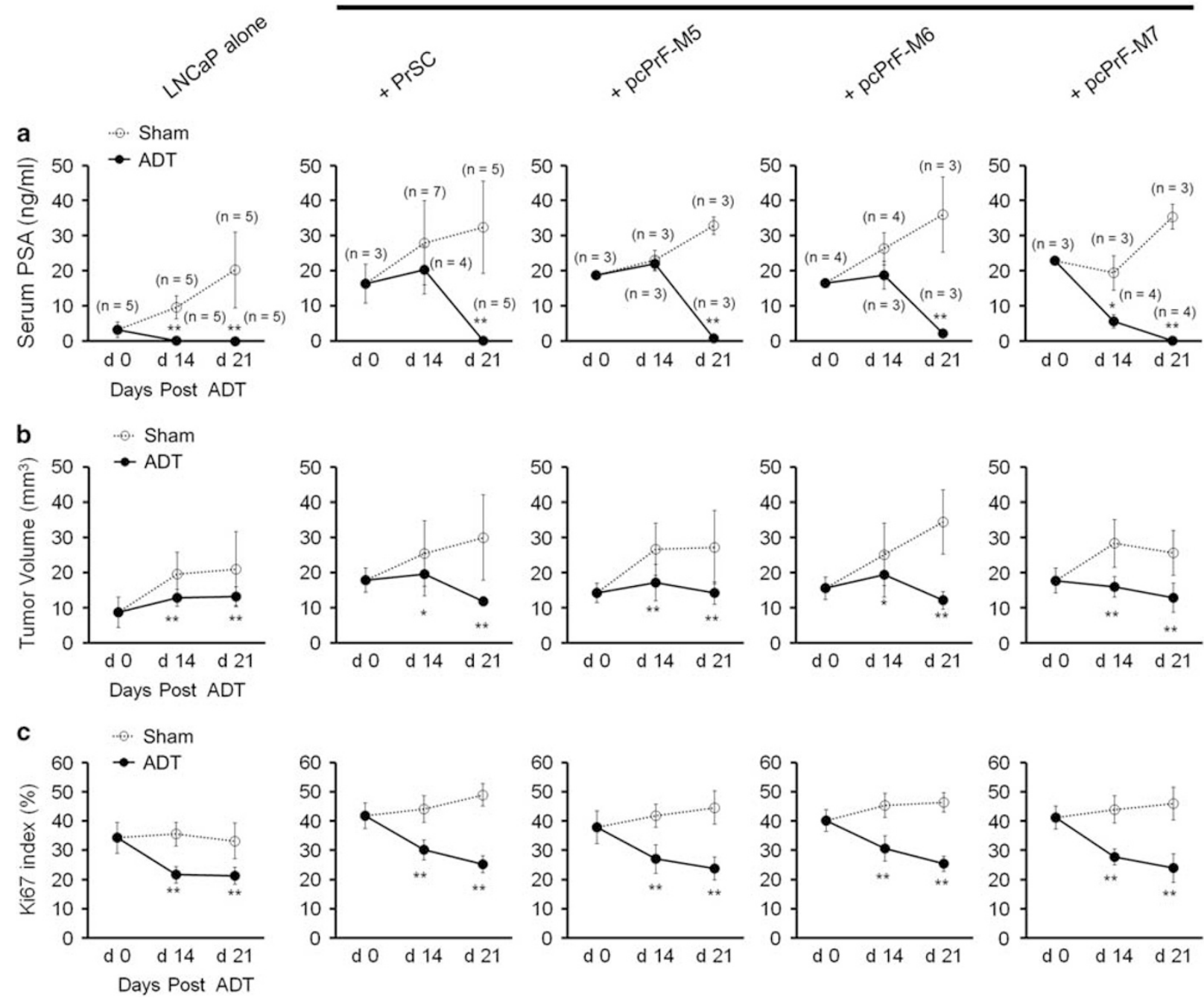

Figure 3 Effects of co-inoculation with fibroblasts on serum PSA decline after ADT in vivo. Changes in serum PSA (a), tumor volume (b), and Ki67 index (c) of LNCaP xenografts were compared in intact or ADT-treated mice among the five groups on days 0,14 , and 21 post $A D T$. ${ }^{*} P<0.05$, ${ }^{* *} P<0.01$ vs sham-operated control. ADT, androgen deprivation therapy; PSA, prostatespecific antigen.

Table 2 MVD changes in LNCaP tumors after ADT

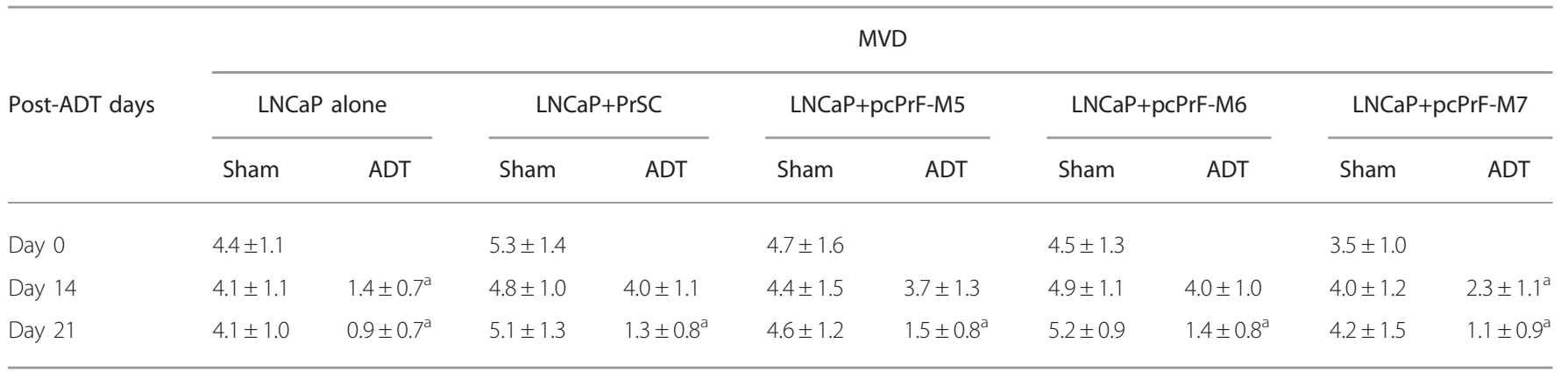




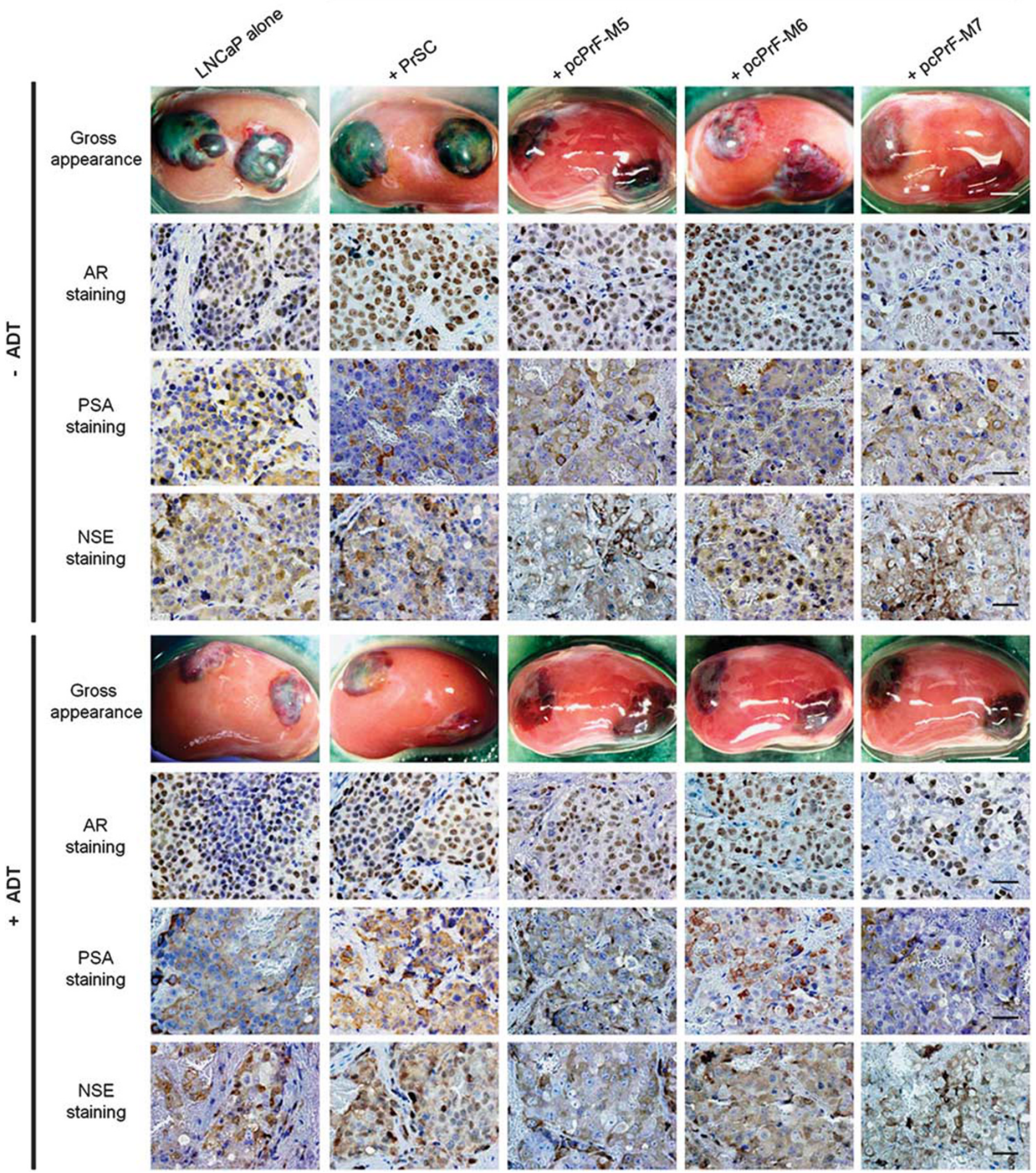

Figure 4 Effects of ADT on histopathological characteristics of xenografts derived from co-inoculation of LNCaP cells with fibroblasts in vivo. (a) Characteristic gross appearances of LNCaP cells alone and LNCaP cells plus fibroblasts in both intact and ADT-treated mice (bar $=2 \mathrm{~mm})$, and representative images of AR, PSA, and NSE staining of mice from each group on day 21 post ADT. Bar $=100 \mu$ m, magnification $=\times 400$. (b) Representative images of MVD staining in both intact and ADT-treated mice from each group on day 14 post ADT. Arrow, CD31-positive vessels with the lumen. Bar $=100 \mu \mathrm{m}$, magnification $=\times 200$. ADT, androgen deprivation therapy; MVD, microvessel density; PSA, prostatespecific antigen. 

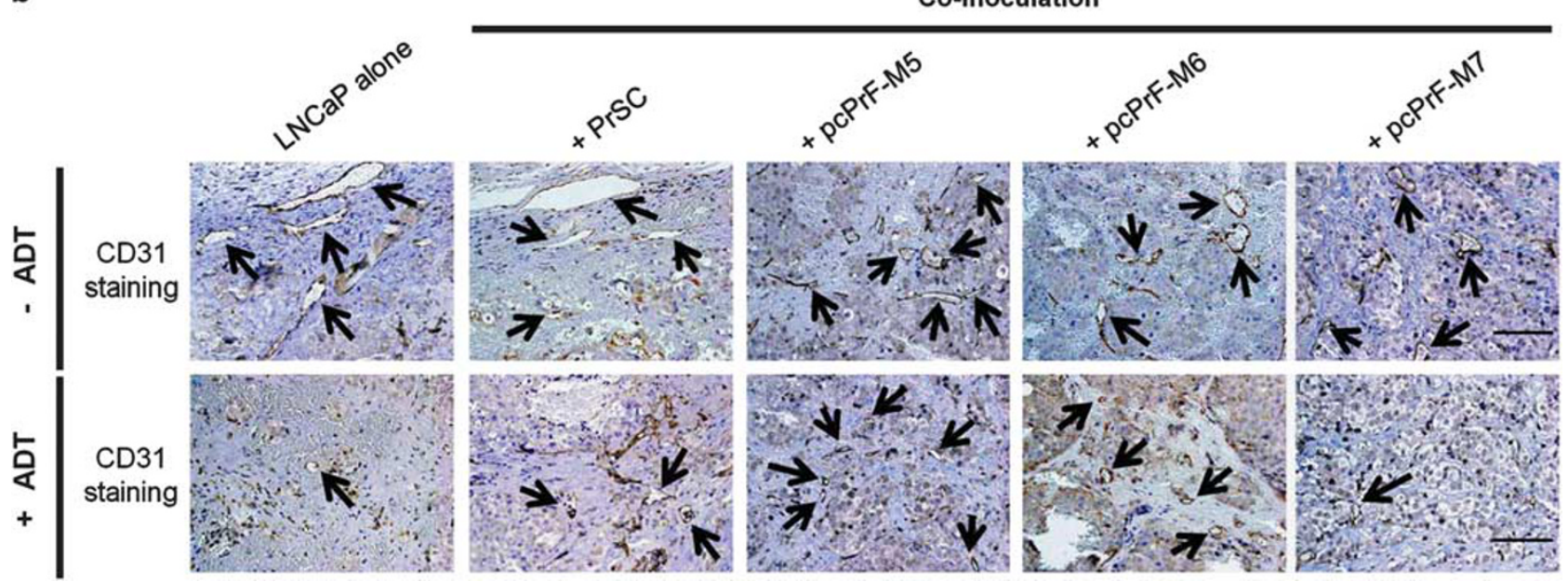

Figure 4 Continued.

a
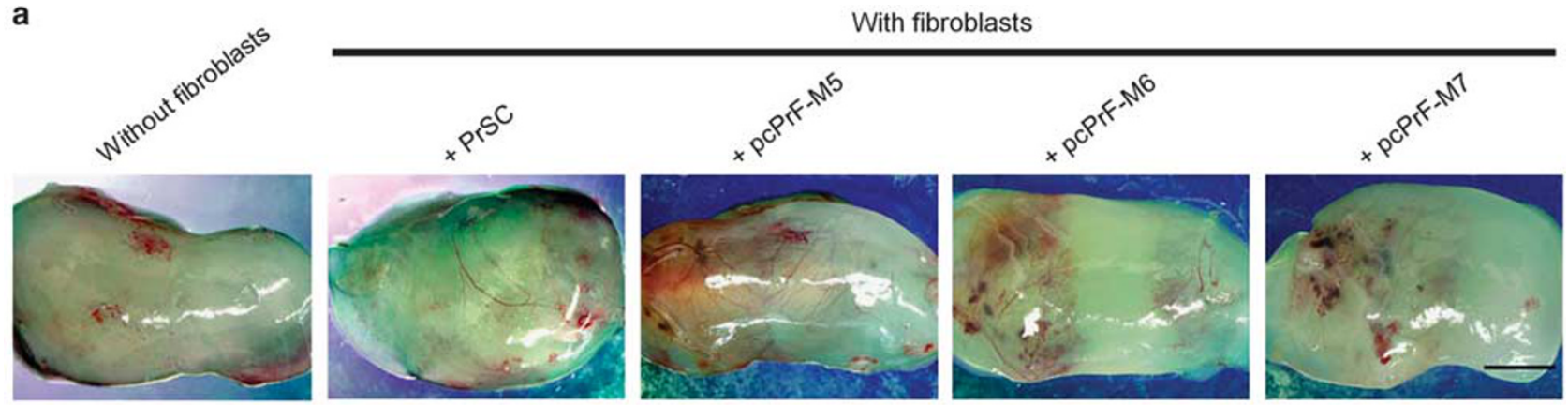

b

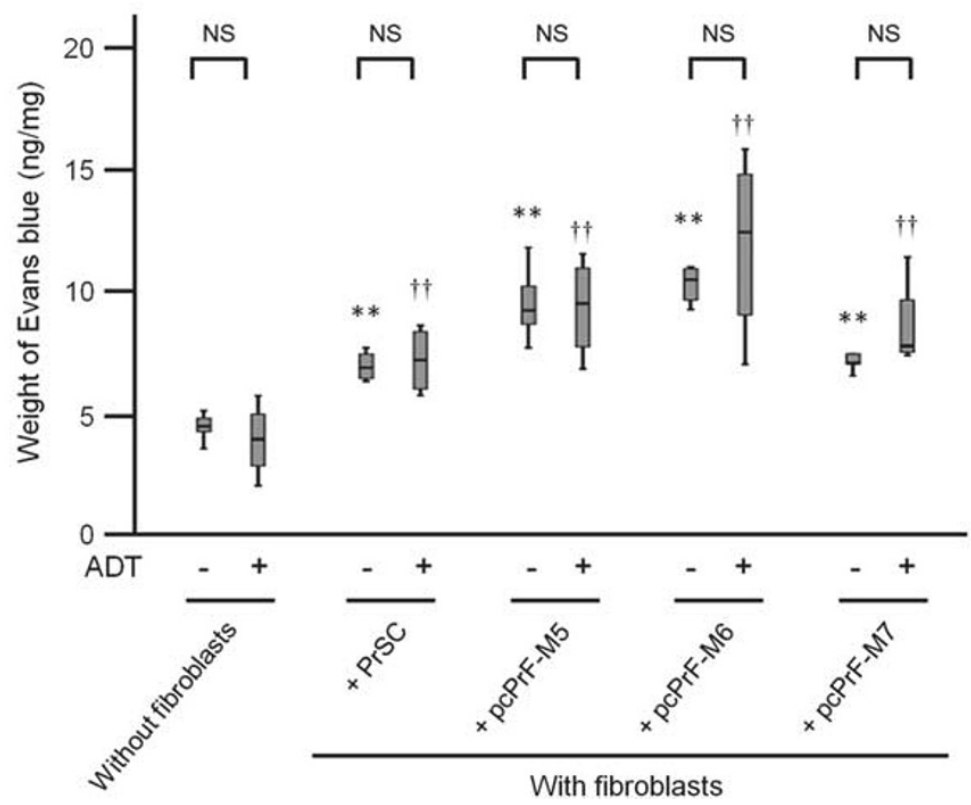

Figure 5 Effects of prostate fibroblasts on angiogenesis in vivo. Bar $=2 \mathrm{~mm}$. (a) Characteristic gross appearance of Matrigel combined with fibroblasts. (b) The amount of Evans blue dye was quantified by $A_{620}$ and normalized to the Matrigel weight. ${ }^{* *} P<0.01$ vs without fibroblasts, ADT ( $)$. ${ }^{\dagger+} P<0.01$ vs without fibroblasts, ADT (+). ADT, androgen deprivation therapy; NS, not significant. 


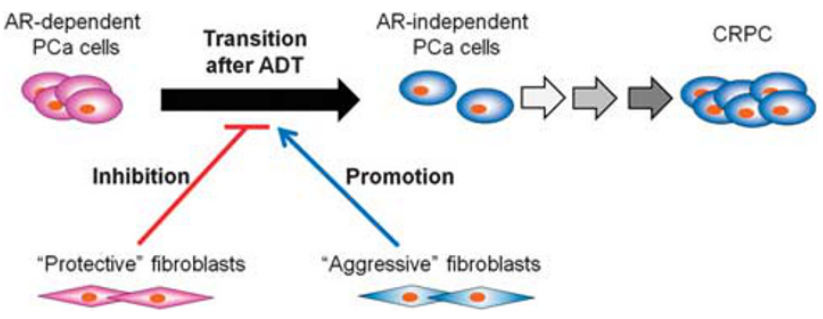

Figure 6 The concept and role of fibroblasts after ADT in PCa patients. 'Protective' fibroblasts inhibit transition of AR-dependent PCa cells to AR independent. 'Aggressive' fibroblasts promote transition of AR-dependent $P C a$ cells to $A R$ independent. ADT, androgen deprivation therapy; $A R$, androgen receptor.

an AR blocker. In contrast, acute loss of AR stimulation in $\mathrm{PCa}$ cells in mice inoculated with LNCaP cells alone may be transformed from an AR-dependent state to an AR-independent state, i.e., stage $4 \mathrm{PCa}$ exhibiting NE differentiation; this stage is considered less responsive to AR blockers. Thus, AR stimulation after ADT may have an important role in maintaining the AR dependence of PCa cells.

In our in vivo analysis, we observed that the tumor structure exhibited marked changes after ADT, which contributed to the decline in serum PSA observed with increased vascularization of tumors. Moreover, even if serum PSA levels were undetectable, AR and PSA proteins were still detected in all tumors. Previous studies have reported that castration has a role in the reduction of blood flow in the rat ventral prostate gland. ${ }^{30,31}$ The reduced number of blood vessels suggests that PSA levels in the circulation may have decreased because of the loss of secreted PSA entering the capillaries in the stroma. Godoy et al focused on paracrine signaling mediated through stromal or epithelial cells, and demonstrated the time course of changes in the vascular structure of tumors after ADT using a human prostate cancer primary xenograft model. ${ }^{32}$ They found that VEGF production by stromal cells has an important role as a mediator of the prostate vasculature. Generally, VEGF, FGF2, and TGF $\beta$ derived from fibroblasts could contribute to neovascularization. ${ }^{33}$ We confirmed that fibroblasts exhibiting abundant secretion of VEGF and TGF $\beta 1$, such as pcPrF-M5 and pcPrF-M6, showed greater neovascularization. Therefore, MVD on day 14 post ADT in mice inoculated with LNCaP cells plus pcPrF-M5 or pcPrF-M6 was maintained compared with that in shamoperated mice. Taken together, these results indicated that heterogeneous angiogenic factors secreted from fibroblasts regulated the reduction in the number of tumor blood vessels after ADT, which was correlated with the decline in serum PSA.

Soluble growth factors and cytokines derived from fibroblasts are known to directly affect AR stimulation and AR-regulated PSA expression in the context of androgen deprivation. The castration-induced decrease in serum PSA following subcutaneous inoculation with LNCaP cells plus human bone fibroblasts as an in vivo model of PCa has been reported previously; ${ }^{34}$ in this study, a correlation between serum PSA levels and three growth factors (ie, FGF2, TGF $\alpha$, and $\operatorname{TGF} \beta$ ) was observed. In addition, Shigemura et al showed that conditioned medium from co-cultures of LNCaP cells and human prostate stromal fibroblasts induced PSA promoter reporter activity, ERK phosphorylation, and AR phosphorylation in LNCaP cells in vitro. ${ }^{35}$ Our investigation revealed that fibroblast heterogeneity directly affected PSA expression and the activation of Stat3, but not Akt or ERK, in LNCaP cells co-cultured in vitro. In addition, previous studies have suggested that IGF1, KGF (also known as FGF7), EGF, and IL6 stimulate AR and PSA expression in the context of androgen deficiency ${ }^{12,36,37}$ via signaling pathways including Akt, Stat3, and ERK. ${ }^{11}$ We confirmed that EGF, IGF1, and IL6 stimulated the expression of AR and PSA proteins in LNCaP cells, suggesting that soluble factors derived from fibroblasts may function similar to androgen in the absence of androgen. Thus, a heterogeneous combination of growth factors and cytokines derived from fibroblasts could be responsible for $\mathrm{AR}$ stimulation of PCa cells in the context of androgen deprivation.

In this study, we formed a hypothesis to explain our previous clinical observations. Tumor-promoting 'aggressive' fibroblasts, so-called cancer-associated fibroblasts (CAFs), are well known. ${ }^{38}$ CAFs surround cancer cells to support the survival and proliferation of cancer cells in a paracrine fashion. On the other hand, Hayashi et al reported that rat urogenital sinus mesenchyme, which had some features similar to CAFs, but that biologically works to promote the development, differentiation and ultimately growth quiescence of the prostate elicited a reduction in tumorigenic potential of Dunning prostatic adenocarcinoma. ${ }^{39}$ Recent studies have also demonstrated that normal human fibroblasts can inhibit the proliferation of tumor cells. ${ }^{40,41}$ We hypothesize that these 'protective' fibroblasts could also preserve the AR dependence of PCa cells after ADT. Moreover, Banerjee et al demonstrated that epigenetic changes in prostatic fibroblasts could cause DNA damage, mediating prostate tumor progression. ${ }^{42}$ Thus, androgen deficiency may contribute to reciprocal transfer of fibroblasts between 'protective' and 'aggressive' states. Although Ayala et al evaluated the possibility of using quantification of reactive stroma elements for prediction of disease progression, ${ }^{43}$ we would suggest that the quality of fibroblasts is also an important factor that can be used to distinguish between 'aggressive' and 'protective' fibroblasts, which may be determined by the combination of stromal biomarkers. Future studies are needed to identify the specific profile of stroma-derived factors responsible for disease progression.

Supplementary Information accompanies the paper on the Laboratory Investigation website (http://www.laboratoryinvestigation.org)

\section{ACKNOWLEDGMENTS}

We are grateful to Professor Simon Hayward of the Department of Cancer Biology, NorthShore University HealthSystem Research Institute for critical reading of the manuscript. We thank Mrs Yumi Yoshikawa and Mrs Izumi Matsuoka for their technical support. This work was supported by Grants-in-Aid from the Ministry of Education for Science and Culture of Japan 
(grant number 26861266), the 2012 Mie Medical Research Foundation, the 2013 Mie Health Care Administration Center Foundation, and the 2013 Mie Medical Association Foundation.

\section{DISCLOSURE/CONFLICT OF INTEREST}

The authors declare no conflict of interest.

1. Hernandez J, Thompson IM. Prostate-specific antigen: a review of the validation of the most commonly used cancer biomarker. Cancer 2004;101:894-904.

2. Fizazi KS, Higano CS, Nelson JB et al. Phase III, randomized, placebocontrolled study of docetaxel in combination with zibotentan in patients with metastatic castration-resistant prostate cancer. J Clin Oncol 2013;31:1740-1747.

3. Crawford ED, Bennett CL, Andriole GL et al. The utility of prostatespecific antigen in the management of advanced prostate cancer. BJU Int 2013;112:548-560.

4. Arai Y, Yoshiki T, Yoshida O. Prognostic significance of prostate specific antigen in endocrine treatment for prostatic cancer. J Urol 1990;144: 1415-1419.

5. Sasaki T, Onishi T, Hoshina A. Nadir PSA level and time to PSA nadir following primary androgen deprivation therapy are the early survival predictors for prostate cancer patients with bone metastasis. Prostate Cancer Prostatic Dis 2011;14:248-252.

6. Sasaki T, Onishi T, Hoshina A. Cutoff value of time to prostate-specific antigen nadir is inversely correlated with disease progression in advanced prostate cancer. Endocr Relat Cancer 2012;19:725-730.

7. Choueiri TK, Xie W, D'Amico AV et al. Time to prostate-specific antigen nadir independently predicts overall survival in patients who have metastatic hormone-sensitive prostate cancer treated with androgendeprivation therapy. Cancer 2009;115:981-987.

8. Kitagawa $\mathrm{Y}$, Ueno $\mathrm{S}$, Izumi $\mathrm{K}$ et al. Nadir prostate-specific antigen (PSA) level and time to PSA nadir following primary androgen deprivation therapy as independent prognostic factors in a Japanese large-scale prospective cohort study (J-Cap). J Cancer Res Clin Oncol 2014;140: 673-679.

9. Yousef GM, Diamandis EP. The new human tissue kallikrein gene family: structure, function, and association to disease. Endocr Rev 2001;22: 184-204.

10. Culig $\mathrm{Z}$, Klocker $\mathrm{H}$, Bartsch $\mathrm{G}$ et al. Androgen receptors in prostate cancer. Endocr Relat Cancer 2002;9:155-170.

11. Zhu ML, Kyprianou N. Androgen receptor and growth factor signaling cross-talk in prostate cancer cells. Endocr Relat Cancer 2008;15:841-849.

12. Kim J, Coetzee GA. Prostate specific antigen gene regulation by androgen receptor. J Cell Biochem 2004;93:233-241.

13. Kato $M$, Ishii $K$, Iwamoto $Y$ et al. Activation of Fgf2-Fgfr signaling in the castrated mouse prostate stimulates the proliferation of basal epithelial cells. Biol Reprod 2013;89:81.

14. Gravina GL, Mancini A, Ranieri G et al. Phenotypic characterization of human prostatic stromal cells in primary cultures derived from human tissue samples. Int J Oncol 2013;42:2116-2122.

15. Tanner MJ, Welliver Jr RC, Chen $\mathrm{M}$ et al. Effects of androgen receptor and androgen on gene expression in prostate stromal fibroblasts and paracrine signaling to prostate cancer cells. PLoS One 2011;6:e16027.

16. Ishii K, Mizokami A, Tsunoda $T$ et al. Heterogenous induction of carcinoma-associated fibroblast-like differentiation in normal human prostatic fibroblasts by co-culturing with prostate cancer cells. J Cell Biochem 2011;112:3604-3611.

17. Ishii K, Imamura $\mathrm{T}$, Iguchi $\mathrm{K}$ et al. Evidence that androgen-independent stromal growth factor signals promote androgen-insensitive prostate cancer cell growth in vivo. Endocr Relat Cancer 2009;16:415-428.

18. Halin $S$, Hammarsten $P$, Wikstrom $P$ et al. Androgen-insensitive prostate cancer cells transiently respond to castration treatment when growing in an androgen-dependent prostate environment. Prostate 2007:67:370-377.

19. Sobel RE, Sadar MD. Cell lines used in prostate cancer research: a compendium of old and new lines-part 2. J Urol 2005;173:360-372.

20. Sobel RE, Sadar MD. Cell lines used in prostate cancer research: a compendium of old and new lines-part 1. J Urol 2005;173:342-359.
21. Krill D, Shuman M, Thompson MT et al. A simple method for the isolation and culture of epithelial and stromal cells from benign and neoplastic prostates. Urology 1997;49:981-988.

22. Webber MM, Trakul N, Thraves PS et al. A human prostatic stromal myofibroblast cell line Wpmy-1: a model for stromal-epithelial interactions in prostatic neoplasia. Carcinogenesis 1999;20: 1185-1192.

23. Mohler JL, Gregory CW, Ford 3rd OH et al. The androgen axis in recurrent prostate cancer. Clin Cancer Res 2004;10:440-448.

24. Titus MA, Schell MJ, Lih FB et al. Testosterone and dihydrotestosterone tissue levels in recurrent prostate cancer. Clin Cancer Res 2005;11: 4653-4657.

25. Iwamoto Y, Ishii K, Sasaki T et al. Oral naftopidil suppresses human renal-cell carcinoma by inducing $\mathrm{G}(1)$ cell-cycle arrest in tumor and vascular endothelial cells. Cancer Prev Res (Phila) 2013;6:1000-1006.

26. Jennbacken $\mathrm{K}$, Tesan $\mathrm{T}$, Wang $\mathrm{W}$ et al. $\mathrm{N}$-Cadherin increases after androgen deprivation and is associated with metastasis in prostate cancer. Endocr Relat Cancer 2010;17:469-479.

27. Nelson PS. Molecular states underlying androgen receptor activation: a framework for therapeutics targeting androgen signaling in prostate cancer. J Clin Oncol 2012;30:644-646.

28. Burchardt $T$, Burchardt $M$, Chen MW et al. Transdifferentiation of prostate cancer cells to a neuroendocrine cell phenotype in vitro and in vivo. J Urol 1999;162:1800-1805.

29. Yuan TC, Veeramani S, Lin FF et al. Androgen deprivation induces human prostate epithelial neuroendocrine differentiation of androgensensitive Lncap cells. Endocr Relat Cancer 2006;13:151-167.

30. Shabsigh A, Chang DT, Heitjan DF et al. Rapid reduction in blood flow to the rat ventral prostate gland after castration: preliminary evidence that androgens influence prostate size by regulating blood flow to the prostate gland and prostatic endothelial cell survival. Prostate 1998;36:201-206.

31. Shabsigh A, Lee B, Buttyan R. Unique morphological aspects of the rat ventral prostate gland revealed by vascular corrosion casting. Prostate 1999:39:240-245.

32. Godoy A, Montecinos VP, Gray DR et al. Androgen deprivation induces rapid involution and recovery of human prostate vasculature. Am J Physiol Endocrinol Metab 2011;300:E263-E275.

33. van Moorselaar RJ, Voest EE. Angiogenesis in prostate cancer: its role in disease progression and possible therapeutic approaches. Mol Cell Endocrinol 2002;197:239-250.

34. Gleave ME, Hsieh JT, Wu HC et al. Serum prostate specific antigen levels in mice bearing human prostate Incap tumors are determined by tumor volume and endocrine and growth factors. Cancer Res 1992;52:1598-1605.

35. Shigemura $\mathrm{K}$, Isotani $\mathrm{S}$, Wang $\mathrm{R}$ et al. Soluble factors derived from stroma activated androgen receptor phosphorylation in human prostate Incap cells: roles of Erk/Map kinase. Prostate 2009;69:949-955.

36. Ueda T, Mawji NR, Bruchovsky $\mathrm{N}$ et al. Ligand-independent activation of the androgen receptor by interleukin- 6 and the role of steroid receptor coactivator-1 in prostate cancer cells. J Biol Chem 2002;277: 38087-38094.

37. Culig Z, Hobisch A, Cronauer MV et al. Androgen receptor activation in prostatic tumor cell lines by insulin-like growth factor-i, keratinocyte growth factor, and epidermal growth factor. Cancer Res 1994;54: $5474-5478$

38. Olumi AF, Grossfeld GD, Hayward SW et al. Carcinoma-associated fibroblasts direct tumor progression of initiated human prostatic epithelium. Cancer Res 1999;59:5002-5011.

39. Hayashi N, Cunha GR. Mesenchyme-induced changes in the neoplastic characteristics of the dunning prostatic adenocarcinoma. Cancer Res 1991;51:4924-4930.

40. Flaberg E, Markasz L, Petranyi G et al. High-throughput live-cell imaging reveals differential inhibition of tumor cell proliferation by human fibroblasts. Int J Cancer 2011;128:2793-2802.

41. Alkasalias $T$, Flaberg $E$, Kashuba $V$ et al. Inhibition of tumor cell proliferation and motility by fibroblasts is both contact and soluble factor dependent. Proc Natl Acad Sci USA 2014;111:17188-17193.

42. Banerjee J, Mishra R, Li X et al. A reciprocal role of prostate cancer on stromal DNA damage. Oncogene 2014;33:4924-4931.

43. Ayala G, Tuxhorn JA, Wheeler TM et al. Reactive stroma as a predictor of biochemical-free recurrence in prostate cancer. Clin Cancer Res 2003;9:4792-4801. 